\title{
Strongly Hyperbolic Systems of Maximal Rank
}

\author{
Dedicated to Professor Yujiro Ohya on his sixtieth birthday
}

By

Tatsuo NISHITANI*

\section{$\S 1$. Introduction}

Let $L$ be a first order differential operator defined in an open set $\Omega$ in $\mathbb{R}^{n+1}$

$$
L(x, D)=D_{0} I_{m}+\sum_{j=1}^{n} A_{j}(x) D_{j}
$$

where $A,(x)$ are real analytic $m \times m$ matrices defined in $\Omega$ and $x=\left(x_{0}, x^{\prime}\right)=$ $\left(x_{0}, x_{1}, \ldots, x_{n}\right), \xi=\left(\xi_{0}, \xi^{\prime}\right)=\left(\xi_{0}, \xi_{1}, \ldots, \xi_{n}\right)$. Let us denote by $h(x, \xi)$ and $M(x, \xi)$ the determinant and the cofactor matrix of $L(x, \xi)$ respectively. Let $\sum=\{z=$ $\left.(x, \xi) \mid h(z)=\cdots=d^{m-1} h(z)=0\right\}$ be the set of characteristics of order $m$ of $h$. We assume that $\sum$ is a real analytic manifold near a reference point $\widehat{z}=(\widehat{x}, \widehat{\xi})$. Without restrictions we may suppose that $0 \in \Omega$ and $\widehat{x}=0$. Let $\sum$ be given by

$$
\phi_{0}(x, \xi)=\xi_{0}=0, \quad \phi_{j}\left(x, \xi^{\prime}\right)=0,1 \leq_{j} \leq_{k}
$$

where $\phi_{j}\left(x, \xi^{\prime}\right)$ are real analytic, homogeneous of degree 0 in $\xi^{\prime}$ with linearly independent differentials at $\widehat{z}$. Since we are interested in strongly hyperbolic systems, we assume that $L(x, \xi)$ satisfies a necessary condition for strong hyperbolicity obtained in [5], that is

$$
M(x, \xi) \text { vanishes of order } m-2 \text { on } \sum
$$

which implies in particular $\left(\left.L\right|_{\Sigma}\right)^{2}=O$ where $\left.L\right|_{\Sigma}$ is the restriction of $L$ to $\Sigma$. Thus we have

\footnotetext{
Communicated by T. Kawa1, December 20, 1996.

1991 Mathematics Sobject Classification: Primary 35M50, 35L45, secondary 35M10.

Key words and phrases. Strongly hyperbolic systems, maximal rank. Cauchy problem.

*Department of Mathematics, Graduate School of Science, Osaka University, Machikaneyama 1-16. Toyonaka Osaka 560, Japan
} 


$$
0 \leq \operatorname{rank}\left(\left.L\right|_{\Sigma}\right) \leq[m / 2] .
$$

In our previous papers [6], [7] we studied the extreme case that $\operatorname{rank}\left(\left.L\right|_{\Sigma}\right)=0$, the case closest to symmetric systems. In this note we study the other extreme case when $\operatorname{rank}\left(\left.L\right|_{\Sigma}\right)=[m / 2]$, which is, in a sense, farthest away from symmetric systems. Our aim is to show that if the localization $h_{z}$ of $h(x, \xi)$ at $z \in \sum$, the first non-trivial term in the Taylor expansion of $h$ at $z$ which is a polynomial on $T_{z}\left(T^{*} \Omega\right) / T_{z} \sum$, is strictly hyperbolic and the propagation cone of $h_{z}$ is transversal to $\sum$ at every $z \in \sum$ then $L(x, D)$ is strongly hyperbolic (Theorem 1.2). Here the propagation cone is defined as the dual cone of the hyperbolic cone of $h_{z}$ with respect to the canonical symplectic structure on $T_{z}\left(T^{*} \Omega\right)$.

We remark that $L$ is not symmetrizable and $h$, as a scalar operator, is not strongly hyperbolic if $m \geq 3$. In fact in order that $h$ is strongly hyperbolic then every characteristic must be at most double ([1]).

The idea of the proof of strong hyperbolicity is very simple. Let $S^{m}(=$ $\left.S_{1,0}^{m}\right)$ denote the space of symbols of order $m$ and denote by $\Psi^{m}$ the space of pseudo-differential operators with symbol in $S^{m}$ (for the definition, see for example [2]). Then we can find $M_{\imath} \in \Psi^{m-1-\imath}$ so that for any lower order $B(x)$ we can apply our previous results in [4], [3], on the well posedness of the Cauchy problem for scalar operators or rather its proof to $(L+B)\left(M+M_{1}+\right.$ $M_{2}$ ).

Theorem $\mathbb{1}_{0} \mathbb{1}$. Let $\widehat{z} \in \sum$ and on $\sum$ near $\widehat{z}$ we assume that $\operatorname{rank} L(x, \xi)=$ $[m / 2]$ and $M(x, \xi)$ vanishes of order $m-2$. Then one can find $M_{i} \in \Psi^{m-1-1}, i=1$, 2 defined near $\widehat{z}$ such that with

$$
(L+B)\left(M+M_{1}+M_{2}\right)=h I_{m}+H_{m-1}+\cdots+H_{m-j}+\cdots
$$

where $H_{m-j} \in \Psi^{m-j}$ near $\widehat{z}$, we have either (1) or (11):

(1) for every $B(x) \in C^{\infty}(\Omega ; M(m, \mathbb{C})), H_{m-1}(x, \xi)$ vanishes of order $m-2 j$ on $\sum$ near $\widehat{z}$,

(11) for every $B(x) \in C^{\infty}(\Omega ; M(m, \mathbb{C}))$ all elements of $H_{m-j}(x, \xi)$ vanish of order $m-2 j$ on $\sum$ near $\widehat{z}$ except for the last row and column which vanishes of order $m-2 j$ -1 and $m-2 j+1$ on $\sum$ near $\widehat{z}$ respectively.

Remark. We can find $M_{1} \in \Psi^{m-2}$ so that either $(L+B)\left(M+M_{1}\right)$ or $\left(M+M_{1}\right)$ $(L+B)$ verifies the assertion $(1)$ of Theorem 1.1. We give the proof at the end of Section 3.

In virtue of Theorem 1.1 we can apply our previous results or rather its proof in [4], [3] to get

Theorem 1.2. Assume the same assumptions as in Theorem 1.1. Suppose that the localization $h_{\hat{z}}$ is strictly hyperblic on $T_{\hat{z}}\left(T^{*} \Omega\right) / T_{\hat{z}} \sum$ and the propagation cone of $h_{\vec{z}}$ is iransversal to $\sum$ at $\widehat{z}$. Then $L$ is microlocally strongly hyperbolic near $\widehat{z}$. 
With $A\left(x, \xi^{\prime}\right)=\sum_{j=1}^{n} A_{j}(x) \xi_{j}$ we can write

$$
A\left(x, \xi^{\prime}\right)=\sum_{j=1}^{k} B_{j}\left(x, \xi^{\prime}\right) \phi_{j}\left(x, \xi^{\prime}\right)+P\left(x, \xi^{\prime}\right)
$$

with $P\left(x, \xi^{\prime}\right)=\left.A\left(x, \xi^{\prime}\right)\right|_{\Sigma}$. Then we have $\operatorname{rank} P\left(x, \xi^{\prime}\right)=[m / 2]$ near $\widehat{z}$ by assumption. Let us write

$$
J(r)=\left(\begin{array}{cccc}
0 & 1 & & \\
& \ddots & \ddots & \\
& & \ddots & 1 \\
& & & 0
\end{array}\right) \in M(r, \mathbf{R})
$$

and denote $J\left(r_{1}, \ldots, r_{s}\right)=\bigoplus_{j=1}^{s} J\left(r_{j}\right)$. The next lemma is well known

Lemma 1.3. Suppose that $\operatorname{rank} P\left(x, \xi^{\prime}\right)=[m / 2]$ near $\widehat{z}$. Then there is a real analytic $N\left(x, \xi^{\prime}\right) \in S^{0}$ defined near $\widehat{z}$ satisfying

$$
N\left(x, \xi^{\prime}\right)^{-1} P\left(x, \xi^{\prime}\right) N\left(x, \xi^{\prime}\right)=J\left|\xi^{\prime}\right|
$$

where $J=\bigoplus_{1}^{[m / 2]} J(2)$ if $m$ is even and $J=\bigoplus_{1}^{[m / 2]} J(2) \oplus\{0\}$ if $m$ is odd.

Since the existence of a parametrix of the Cauchy problem with finite propagation speed, which assures the well posedness of the Cauchy problem, is independent of changes of basis for $\mathbb{C}^{m}$, one can assume that

$$
A\left(x, \xi^{\prime}\right)=\sum_{j=1}^{k} B_{j}\left(x, \xi^{\prime}\right) \phi_{j}\left(x, \xi^{\prime}\right)+J\left|\xi^{\prime}\right|
$$

if rank $A\left(x, \xi^{\prime}\right)=[m / 2]$ near $\widehat{z}$ where $B_{j} \in S^{1}$ is real analytic near $\widehat{z}$ and $J$ is given in Lemma 1.3 .

\section{§2. Lemmas}

Let

$$
L(x, \xi)=\left(a_{2}(x, \xi)\right)_{1 \leq \imath, \jmath \leq m}=\xi_{0} I_{m}+\sum_{j=1}^{k} B_{j}\left(x, \xi^{\prime}\right) \phi,\left(x, \xi^{\prime}\right)+J\left|\xi^{\prime}\right|
$$

where $B_{j}$ are real analytic near $\widehat{z} . \quad$ Let $J=\bigoplus_{1}^{s} J(2) \bigoplus_{s+1}^{t}\{0\}$ with $m=2 s+t$. We actually interested in the case $t=s+1$. Denote by $\tilde{a}_{i j}$ the cofactor of $a_{i j}$ in $L(x, \xi)$ so that $M=\left(\tilde{a}_{j_{l}}\right)$, the transposed of $\left(\tilde{a}_{i j}\right)$. We denote $C(x, \xi)=O(s)$ if $C(x, \xi)$ vanishes of order $s$ on $\sum$ near $\widehat{z}$ and we write $C(x, \xi)=O_{w}(s)$ if $C(x, \xi)$ vanishes of order $s$ at $w \in \sum$. Note that

$$
a_{i j}=O(1) \text { unless }(i, j)=(2 k-1,2 k), \quad 1 \leq k \leq s .
$$


In this section we show

Lemma 2.1. Assume that $h=O(m), M=O(m-2)$. Then we have

$$
a_{2 i, 2 j-1}=O(2), \quad 1 \leq i, j \leq s .
$$

We first observe that

Lemmma 2.2. Assume that $M(x, \xi)=O(m-2)$. Then we have

$$
\begin{aligned}
& a_{2 k-2 l+2,2 k-1}=O(2), \quad 1 \leq k \leq s, \quad 2 \leq l, \\
& a_{2 k, 2 k-2 l+1}=O(2), \quad 1 \leq k \leq s, \quad 2 \leq l .
\end{aligned}
$$

In particular, $a_{2 \imath, 2 \jmath-1}=O(2), 1 \leq i, j \leq s, i \neq j$.

Proof. Let us denote by $S_{m}$ the set of all permutations on $\{1,2, \ldots, m\}$. Note that

$$
\tilde{a}_{2 k, 2 k-2 l+1}=\cdots+\sum_{\sigma \in T} a^{\sigma} \xi_{0}^{m-4}+\cdots=O(m-2), a^{\sigma}=\prod_{\imath \neq 2 k, \sigma(\imath) \neq \imath} a_{\imath \sigma(\imath)}
$$

where $T=\left\{\sigma \in S_{m} \mid \#\{i \mid \sigma(i)=i\}=m-4, \sigma(2 k)=2 k-2 l+1\right\}$. We show that $a^{\sigma}=O(2)$ if $\sigma \in T$ unless $\sigma(2 k-2 l+1)=2 k-2 l+2, \sigma(2 k-1)=2 k$ and hence $a^{\sigma}= \pm a_{2 k-2 l+2,2 k-1}+O(2)$ which proves the assertion. Let $1 \leq_{k} \leq_{s}$ and $\sigma \in T$. Assume that $\sigma(2 k-2 l+1)=p \neq 2 k-2 l+2$. If $p=2 k$ there is $i$ with $\sigma(i)<i$ and then $a_{2 \sigma(\imath)} a_{2 k-2 l+1,2 k}=O(2)$. If $p \neq 2 k$, with $\sigma(p)=q, \sigma(q)=r$ we have $q \neq 2 k$ if $\sigma \in T$. Thus $p \neq q, q \neq r$ hence $a_{p, q} a_{q, r}=O(1)$ and therefore $a_{2 k-2 l+1, p} a_{p, q} a_{q, r}=$ $O(2)$. Thus we have $a^{\sigma}=O(2)$.

We next assume that $\sigma(2 k-2 l+1)=2 k-2 l+2, \sigma(2 k-1)=p \neq 2 k$. With $\sigma(p)=q, \sigma(q)=r$ we see $p \neq 2 k-1, q \neq 2 k$ if $\sigma \in T$. Thus $p \neq q, q \neq r$ and hence $a_{p, q} a_{q, r}=O(1)$. Then as above we have $a_{2 k-1, p} a_{p, q} a_{q, r}=O(2)$ and hence the assertion. The second assertion can be proved similarly considering $\tilde{a}_{2 k-2 l+2,2 k-1}$.

To complete the proof of Lemma 2.1 it is enough to show

Lemma 2.3. Assume that $h=O(m)$ and $a_{2 t, 2 \jmath-1}=O(2), 1 \leq i, j \leq s, i \neq j$. Then we have

$$
a_{21,2 \jmath-1}=O(2), \quad 1 \leq i, j \leq s .
$$

Proof. It is enough to prove $a_{2 t, 2 \imath-1}=O_{w}(2)$ for every $w \in \sum$ near $\widehat{z}, 1 \leq i$ $\leq s$. Suppose that the assertion does not hold and hence the differentials of $a_{2 i p, 2 \imath p-1}$ at $w \in \sum$ were different from zero for $p=1, \ldots, l, 1 \leq i_{p} \leq s$ and $a_{2,2 t-1}=$ $O_{w}(2)$ if $i \notin\left\{i_{1}, \ldots, i_{l}\right\}$. Set $T=\left\{\sigma \in S_{m} \mid \#\{i \mid \sigma(i)=i\}=m-2 l\right\}$ and $J^{\sigma}=\{1, \ldots, m\}$ $\backslash\{i \mid \sigma(i)=i\}$ for $\sigma \in T$. We recall that

$$
h=\cdots+\sum_{\sigma \in T} a^{\sigma} \xi_{0}^{m-2 l}+\cdots=O(m), \quad a^{\sigma}=\prod_{i, \sigma(\imath) \neq t} a_{\imath \sigma(\imath)}
$$


by assumption. Let $\nu \in T$ be such that $\nu\left(2 i_{p}-1\right)=2 i_{p}, \nu\left(2 i_{p}\right)=2 i_{p}-1$ and note that $a^{\nu}$ vanishes at $w$ exactly of order $l$. We show that $a^{\sigma}=O_{w}(l+1)$ unless $\sigma=\nu$. If $\#\{i \mid \sigma(2 i-1)=2 i\}<l$ then it is clear that $a^{\sigma}=O_{w}(l+1)$. Thus it suffices to consider the case $J^{\sigma}=\left\{2 j_{p}-1,2 j_{p}\right\}_{p=1}^{l}$. Suppose that $J^{\sigma} \neq J^{\nu}$ and hence there were $2 q-1$ such that $2 q-1 \in J^{\sigma}, 2 q-1 \notin J^{\nu}$. Since $a_{2 q, 2 q-1}=O_{w}(2)$ by assumption we get $a^{\sigma}=O_{w}(l+1)$ and hence the assertion. Therefore from (2.2) we would have $a^{\nu}=O_{w}(l+1)$ which is a contradiction.

\section{§3. Proof of Theorem 1.1}

In this section we work near $\widehat{z}$ without mention it. We denote by $\sigma(M)$ the symbol of an operator $M$ and by $O p(M)$ the operator with symbol $M$. But we frequently use $M$ to denote both an operator and its symbol if this leads no confusion.

We first assume that $m$ is even and we write $2 m$ instead of $m$.

Proposition 3.1. Assume that $h(x, \xi)$ and $M(x, \xi)$ vanishes of order $2 m$ and $2 m-2$ on $\sum$ respectively. Then there is $M_{1} \in \Psi^{2 m-2}$ with $\sigma\left(M_{1}\right)=O(2 m-3)$ such that for any $B(x)$ we have

$$
(L+B)\left(M+M_{1}\right)=h I_{2 m}+H_{2 m-1}+\cdots+H_{2 m-\jmath}+\cdots
$$

where $H_{2 m-\jmath} \in \Psi^{2 m-\jmath}$ and $\sigma\left(H_{2 m-\jmath}\right)=O(2 m-2 j)$.

For the proof we remark that

Lemma 3.2. Under the same assumptions as in Proposition 3.1 every even row and odd column of $M$ is $O(2 m-1)$.

Proof. Since $L M=h I_{2 m}$ it follows that $\left\{J\left|\xi^{\prime}\right|+O(|\phi|)\right\}_{M}=h I_{2 m}$ that is $J\left|\xi^{\prime}\right| M$ $=O(2 m-1)$. This implies clearly that every even row of $M$ is $O(2 m-1)$. Considering $M L=h I_{2 m}$ the second assertion follows similarly.

Proof of Proposition 3.1. Recall that $\sigma(L M)=h I_{2 m}+\sum_{\alpha} L^{(\alpha)} M_{(\alpha)} / \alpha$ ! and note that

$$
S^{2 m-\jmath} \ni \sum_{|\alpha|=j} \frac{1}{\alpha !} L^{(\alpha)} M_{(\alpha)}=O(2 m-2-j) .
$$

Since $2 m-2 j \leq 2 m-2-j$ for $j \geq 2$, it is clear that $L M-O p\left(\sum_{|\alpha|=1} L^{(\alpha)} M_{(\alpha)}\right)$ verifies the desired properties. Set

$$
\sum_{|\alpha|=1} L^{(\alpha)} M_{(\alpha)}=T_{e}+T_{o}
$$

where $T_{e}$ and $T_{o}$ consists of even and odd rows of $\sum_{|\alpha|=1} L^{(\alpha)} M_{(\alpha)}$ respectively. Set 


$$
M_{1}=-{ }^{t} J T_{0} \mid \xi^{\prime-1}
$$

so that $J\left|\xi^{\prime}\right| M_{1}=-T_{o}$ and $M_{1}=O(2 m-3)$. Then it follows that $L\left(M+M_{1}\right)=T_{e}+$ desired form, because $L=J\left|\xi^{\prime}\right|+O(|\phi|)$. It is also clear that $B\left(M+M_{1}\right)$ has the desired form for every $B$. Thus it suffices to study $T_{e}$. Note that the $2 i$-th row of $T_{e}$ is given by a sum of the following terms over $|\alpha|=1$

$$
\sum_{k=1}^{2 m} a_{2 i, k}^{(\alpha)} \widetilde{a_{j k}(\alpha)}=\sum_{k: \text { even }} a_{a_{i, k}(\alpha)}^{(\alpha)} \widetilde{a}_{j k(\alpha)}+\sum_{k: \text { odd }} a_{2 i, k}^{(\alpha)} \widetilde{a}_{j k(\alpha)} .
$$

By Lemma 3.2 we see that $\tilde{a}_{j k}=O(2 m-1)$ if $k$ is even and by Lemma 2.1 we have $a_{2 l, k}=O(2)$ if $k$ is odd. Then it follows that $T_{e}=O(2 m-2)$. This proves the assertion.

We turn to the odd $m$ case. We write $2 m+1$ instead of $m$. Our aim is to prove that

Proposition 3.3. Assume that $h(x, \xi)$ and $M(x, \xi)$ vanishes of order $2 m+1$ and $2 m-1$ on $\sum$ respectively. Then we have either ( 1 ) or (11):

(1) there is $M_{1} \in \Psi^{2 m-1}$ with $\sigma\left(M_{1}\right)=O(2 m-2)$ such that

$$
(L+B)\left(M+M_{1}\right)=h I_{2 m+1}+H_{2 m}+\cdots+H_{2 m+1-1}+\cdots
$$

where $H_{2 m-j} \in \Psi^{2 m+1-s}$ and $\sigma\left(H_{2 m+1-\jmath}\right)=O(2 m+1-2 j)$,

(i1) there are $M_{i} \in \Psi^{2 m-1}, i=1,2$ such that

$$
(L+B)\left(M+M_{1}+M_{2}\right)=h I_{2 m+1}+H_{2 m}+\cdots+H_{2 m+1-j}+\cdots
$$

where every element of $\sigma\left(H_{2 m+1-\jmath}\right)$ is $O(2 m+1-2 j)$ except for the last row and column which is $O(2 m-2 j)$ and $O(2 m+2-2 j)$ respectively.

To prove the proposition we start with

Lemman 3.4. Assume that $h(x, \xi)$ and $M(x, \xi)$ vanishes of order $2 m+1$ and $2 m-1$ respectively. Then every even row of $M$ is $O(2 m)$ and every odd column except for the last one is $O(2 m)$.

Proof. The proof is a repetition of that of Lemma 3.2.

Lemma 3.5. Assume that $M(x, \xi)$ vanishes of order $2 m-1$ on $\sum$. If there is $i$ such that $d a_{2 m+1,21-1}(w) \neq 0$ with some $w \in \sum$ near $\widehat{z}$ then we have $\widetilde{a}_{2 m+1, j}=$ $O(2 m)$ for every $j, \widetilde{a_{2 m+1,2 i}}=O(2 m+1)$ for $i \leq m$ and $a_{2 j, 2 m+1}=O(2)$ for $j \leq m$.

Proof. Note that

$$
\begin{aligned}
\sum_{k=1}^{2 m+1} \tilde{a}_{k j} a_{k, 2 i-1}= & \sum_{k: \text { odd }, k \neq 2 m+1} \tilde{a}_{k j} a_{k, 2 l-1}+\sum_{k: \text { even }} \tilde{a}_{k j} a_{k, 21-1} \\
& +\tilde{a}_{2 m+1, j} a_{2 m+1,2 t-1}
\end{aligned}
$$


Recall that $a_{k, 2 i-1}=O(2)$ if $k$ is even and $i \leq m$ by Lemma 2.1 and $\tilde{a}_{k j}=O(2 m)$ if $k \neq 2 m+1$ is odd by Lemma 3.4. From (3.1) we get $\tilde{a}_{2 m+1, j} a_{2 m+1,2 t-1}=O(2 m+$ 1) for every $j$. Since $\tilde{a}_{2 m+1, j}$ is, up to term $O\left(|\phi|^{2 m}\right)$, a polynomial in $\phi$ of degree $2 m-1$ with coefficients which are real analytic on $\sum$ we conclude from (3.1) that the coefficient vanishes near $w$ and so does near $\widehat{z}$. Thus we get the first assertion. To prove the second assertion we note that

$$
\sum_{k=1}^{2 m+1} a_{2 \imath-1, k} \tilde{a}_{2 m+1, k}=0
$$

and $a_{2 t-1,2 i}=\left|\xi^{\prime}\right|, a_{2 t-1, k}=O(1)$ if $k \neq 2 i$. Since $\widetilde{a}_{2 m+1, k}=O(2 m)$ by the first assertion we get the second assertion from (3.2).

We turn to the third assertion. Consider

$$
\begin{gathered}
\sum_{k: \text { odd }, k \neq 2 m+1} a_{2 j, k} \widetilde{a}_{2 m+1, k}+\sum_{k: \text { even }} a_{2 \jmath, k} \widetilde{a}_{2 m+1, k} \\
+a_{2 \jmath, 2 m+1} \widetilde{a}_{2 m+1,2 m+1}=0 .
\end{gathered}
$$

If $k$ is even it follows from the second assertion that $\tilde{a}_{2 m+1, k}=O(2 m+1)$. On the other hand we have $\widetilde{a}_{2 m+1, k}=O(2 m)$ from the first assertion and $a_{2 \jmath, k}=O(2)$ if $k \neq 2 m+1$ is odd by Lemma 2.1. Since $\widetilde{a}_{2 m+1,2 m+1}$ vanishes at $\widehat{z}$ exactly of order $2 m$ the third assertion follows from $(3,3)$.

Lemma 3.6. Assume that $a_{2 m+1,2 i-1}=O(2)$ for every $i \leq m$. Then we have $\tilde{a}_{, 2 m+1}=O(2 m)$ for every $j$.

Proof. Note that

$$
\begin{aligned}
\sum_{k=1}^{2 m+1} a_{2 m+1, k \tilde{a_{j k}}}= & \sum_{k: \text { odd }, k \neq 2 m+1} a_{2 m+1, k} \tilde{a}_{j k}+\sum_{k: \text { even }} a_{2 m+1, k} \widetilde{a}_{j k} \\
& +a_{2 m+1,2 m+1} \widetilde{a_{j, 2 m+1}}=O(2 m+1) .
\end{aligned}
$$

If $k \neq 2 m+1$ is odd then $a_{2 m+1, k}=O(2)$ by assumption. If $k$ is even then $\widetilde{a}_{j k}=$ $O(2 m)$ by Lemma 3.4 . Since $a_{2 m+1,2 m+1}$ vanishes at $\widehat{z}$ exactly of order 1 , the assertion follows from (3.4).

Proof of Proposition 3.3. We divide the proof into two cases.

(a) Assume that $a_{2 m+1,2 i-1}=O(2), i \leq m$. Set

$$
S_{j}=\sum_{|\alpha|=j} L^{(\alpha)} M_{(\alpha)} / \alpha !=T_{j e}+T_{\jmath o}
$$

where $T_{j e}$ consists of even rows and $(2 m+1)$-th row of $S_{j}$ while $T_{j o}$ consists of $(2 i-1)$-th rows of $S_{j}, 1 \leq i \leq m$. Consider the $(2 m+1)$-th row of $S_{1}$ which is a sum of the following terms over $|\alpha|=1$ 


$$
\begin{gathered}
\sum_{k=1}^{2 m+1} a_{2 m+1, k}^{(\alpha)} \tilde{a}_{j k(\alpha)}=\sum_{k: \text { odd }, k \neq 2 m+1} a_{2 m+1, k}^{(\alpha)} \tilde{a}_{j k(\alpha)}+\sum_{k: \text { even }} a_{2 m+1, k}^{(\alpha)} \tilde{a}_{j k(\alpha)} \\
+a_{2 m+1,2 m+1}^{(\alpha)} \tilde{a}_{j, 2 m+1(\alpha) .}
\end{gathered}
$$

From the assumption it follows that $a_{2 m+1, k}^{(\alpha)}=O(1)$ if $k \neq 2 m+1$ is odd. On the other hand from Lemma 3.4 we get $\tilde{a}_{j k(\alpha)}=O(2 m-1)$ if $k$ is even and by Lemma 3.6 we have $\tilde{a}_{, 2 m+1(\alpha)}=O(2 m-1)$ for every $j$. This proves $T_{1 e}=O(2 m-1)$. As in the proof of Proposition 3.1 we set

$$
M_{1}=-{ }^{t} J T_{1 o}\left|\xi^{\prime}\right|^{-1} \text {. }
$$

Then we have $M_{1} \in \Psi^{2 m-1}$ with $M_{1}=O(2 m-2)$ and setting

$$
(L+B)\left(M+M_{1}\right)=h I_{2 m+1}+H_{2 m}+\cdots+H_{2 m+1-\jmath}+\cdots
$$

it is easy to see that $H_{2 m+1-\jmath} \in \Psi^{2 m+1-j}$ and $H_{2 m+1-\jmath}=O(2 m+1-2 j)$ since $L$ has the form $J\left|\xi^{\prime}\right|+O(|\phi|)$.

(b) Assume that there is $i$ such that $d a_{2 m+1,2 t-1}(w) \neq 0$ with some $w \in \sum$ near $\widehat{z}$. Let $S_{j}, T_{j e}$ and $T_{j o}$ be as in (a) again. From Lemmas $2.1,3.4$ and 3.5 we see that $a_{2 \imath, k}=O(2)$ for odd $k$ and $\widetilde{a}_{\jmath k}=O(2 m)$ for even $k$. This proves that

$$
\text { even row of } S_{j}=O(2 m+1-2 j), j=1,2
$$

while the last row of $S_{j}$ is $O(2 m-1-j), j=1,2$. Moreover by Lemma 3.5 we have $\widetilde{a}_{2 m+1, k}=O(2 m)$ for every $k, \widetilde{a}_{2 m+1, k}=O(2 m+1)$ for even $k$ and $a_{2 l, k}=O(2)$ for odd $k$ by Lemmas 2.1 and 3.5. This proves that

$$
\sum_{k=1}^{2 m+1} a_{2 l, k}^{(\alpha)} \widetilde{a}_{2 m+1, k(\alpha)}=O(2 \mathrm{~m}+2-2|\alpha|)
$$

and hence the last column of $T_{\jmath}$ is $O(2 m+2-2 j), j=1,2$. Let us set

$$
M_{j}=-{ }^{t} J T_{j o}\left|\xi^{\prime}\right|^{-1}, \quad j=1,2
$$

so that $M_{j} \in \Psi^{2 m-s}$ and $M_{j}=O(2 m-1-j)$. We remark that the last column of $M$, is $O(2 m-j)$ for $j=0,1,2$ with $M_{0}=M$ because $\widetilde{a}_{2 m+1, k}=O(2 m)$.

Consider $(L+B)\left(M+M_{1}+M_{2}\right)$. Note that $S_{j}=O(2 m+2-2 j)$ if $j \geq 3$ and hence $L M=T_{1}+T_{2}+$ desired form ' ${ }^{\prime 11}$ ). Set

$$
(L+B)\left(M_{1}+M_{2}\right)=-T_{1 o}-T_{2 o}+F_{2 m}+\cdots+F_{2 m+1-3}+\cdots
$$

where $F_{2 m+1-} \in \Psi^{2 m+1-\jmath}$. It is not difficult to see that $F_{2 m+1-j}=O(2 m+1-2 j)$ and the last column of $F_{2 m+1-}$ is $O(2 m+2-2 j)$. This proves the assertion (11).

Finally we give a proof of Remark in Section 1. Since the last column of $M$, is $O(2 m-j)$ and $L=J\left|\xi^{\prime}\right|+O(|\phi|)$ it is enough to prove that, in case (b) above, one can find $M_{1} \in \Psi^{2 m}$ so that $\left(M+M_{1}\right)(L+B)$ verifies the assertion (1) of Proposition 3.1. From Lemma 3.5 it follows that $\tilde{a}_{2 m+1,3} O(2 m)$ and $a_{2 j, 2 m+1}$ $=O(2)$. Let 


$$
S_{1}=\sum_{|\alpha|=1} M^{(\alpha)} L_{(\alpha)}=T_{e}+T_{o}
$$

where $T_{e}$ and $T_{o}$ consists of even and odd columns of $S_{1}$ respectively. Set

$$
M_{1}=-T_{e}^{t} J\left|\xi^{\prime}\right|^{-1}
$$

so that $M_{1} J\left|\xi^{\prime}\right|=-T_{e}$ and $M_{1}=O(2 m-2)$. Then it is clear that $\left(M+M_{1}\right)(L+B)$ $=T_{o}+$ desired term $(:)$. Thus it is enough to study $T_{o}$. Consider

$$
\sum_{k: \text { odd }, k \neq 2 m+1} \widetilde{a}_{k \jmath}^{(\alpha)} a_{k, 2 t+1(\alpha)}+\sum_{k: \text { even }} \widetilde{a}_{k \jmath}^{(\alpha)} a_{k, 21+1(\alpha)}+\widetilde{a}_{2 m+1, j}^{(\alpha)} a_{2 m+1,2 i+1(\alpha)}
$$

From Lemma 3.4 we have $\widetilde{a}_{k J}=O(2 m)$ if $k \neq 2 m+1$ is odd while $a_{k, 2 t-1}=O(2)$ if $k$ is even and $i \leq m$ which follows from Lemma 2.1. Then (3.5) with $|\alpha|=1$ shows that $T_{o}=O(2 m-1)$ except for the last column. We treat the last column which is a sum of (3.5) with $i=m$ over $|\alpha|=1$. Since $a_{k, 2 m+1}=O(2)$ by virtue of Lemma 3.5 the same arguments as above show $T_{0}=O(2 m-1)$ and hence the result.

\section{References}

[1] Ivrı, V. Ya. and Petkov, V. M.. Necessary conditions for the Cauchy problem for non-strictly hyperbolıc equations to be well posed, Uspekh Mat. Nuık. 29 (1974), 3-70.

[2] Hormander. L., The Analysis of Linear Partial Differentual Operators III . Springer-Verlag, Berlin-Herdelberg. 1985

[3] Kajitanı, K., Wakabayashı, S. and Nishıtanı, T, The Cauchy problem for inyperbolic operators of strong type, Duke Math. J., 75 (1994), 357-408.

[4] Nishitanı, T., Hyperbolıc operators with symplectıc multiple characteristics, J. Muth., Kyoto Univ., 29 (1989), 405-447.

[5] Necessary conditions for strong hyperbolicity of first order systems, J. Analyse Muth.. 61 (1993). 181-229.

[6] - On localizations of a class of strongly hyperbolic systems, Osaka J. Math.. 32 (1995), 41-69.

[7] Symmetrization of hyperbolic systems with non-degenerate characteristics, $J$. Funct. Anal.. 132 (1995), 215-272. 
